\title{
WHY IS EPIGENETICS IMPORTANT IN PAEDIATRICS?
}

\author{
Sorin Buzinschi \\ Faculty of Medicine, Transilvania University, Brasov
}

\begin{abstract}
The understanding of the pathological changes in the last decades has become possible due to the epigenetic achievements. This branch of genetics explains the way in which the environment influences the dynamic functioning of genes. The epigenetic variations are determined by nutrition, infections, chemical noxae, and stress. The metabolic programming initiated in the fetal life is decisive in chronic pathology of the adulthood. Infections, allergic diseases, malignant diseases display a significant epigenetic dimension. By acting at the beginning of the life cycle, the paediatrician can intervene in the development of these processes through prophylactic approach and epigenetic therapy, the latter being underway.
\end{abstract}

Keywords: paediatrics, epigenetics, programming, therapy

During the last decades, rudimentary observation indicates a fulminant boost in the incidence of some formerly rare diseases. Explaining the genetic origin confronts with the acknowledgement that the genetic background of the populations is stable, while the populations display major pathological changes due to modifications of the living environment. An overall view of pathology places paediatrics at the beginning of a series of changes which amplify during the lifetime. The global comprehension of the age pathology is burdened by the division into branches of the medical profession, resulting from the enormous quantity of information of each specialization, each of them with a well-defined area and (often) without overlapping other fields of study. The epigenetic approach blurs the formal borders set by the separation into branches and age distribution, starting from the reality of a biological continuity from the embryonic stage to death. In order to adequately understand the dynamic functioning of the genes, we believe it is necessary to include a glossary of epigenetic terms (1).

\section{Epigenetics}

Epigenetic is a branch of genetics which studies the alterations of the phenotypical traits induced by environment through the reversible change in gene functions, without modifying the DNA sequences. Epigenetic regulation is mediated by DNA meth- ylation, physical changes in the structure of the chromatin and by the action of the not coded RNA molecules.

\section{Chromatin}

The complex formed by DNA and histones which comprise chromosomes representes the chromatin. Condensed chromatin (heterocromatin), in which DNA and histones are tightly packed, blocks the access of transcription factors to gene expression. The open chromatin conformation allows transcriptional factors to access DNA and to initiate the transcription.

\section{DNA Methylation}

Methylation of the DNA is the binding of the methyl group to position 5 of the cytosine pyrimidine (C) ring of a cytosine-guanine dinucleotide $(\mathrm{CpG})$, reaction catalysed by DNA metyl-transferases (DNMTs). DNA methylation is an epigenetic process decisive for the repression of transcription through interrupting the binding of transcription factors.

\section{Histone Modifications}

Histone Modifications take place in areas specific through acetylation, methylation and other processes. They modulate gene expression via changes in chromatin structure. Like the DNA methylation, histone modifications are dynamic and actively regulated by a host of catalytic enzymes.

Corresponding author:

Sorin Buzinschi, Faculty of Medicine, Transilvania University, 29 Eroilor Boulevard, Brasov

E-mail: buzinschis@gmail.com 


\section{Genomic Imprinting}

An epigenetic process through which only a single allele from one of the parents is expressed represents the genomic imprinting. It is a fundamental process for growth and development.

\section{Epigenetic Inheritance}

The genetic information mostly transmits from parents to children through the genetic code, but some epigenetic characteristics also maintain and pass from one generation to the next.

\section{Alleles}

Alleles are genes located on chromosomes, each gene occupying a locus. In loci set in the same position on the homologous pair of chromosome, they may be identical versions of the gene (homozygous organism) or different DNA sequences-alleles (heterozygous organism). (Fig. 1)

The way in which gene differently express, without DNA sequence alteration, is determined by their functional changes through DNA methylation, histone and microRNA modifications, the genetic background being regarded as the "hard" component and epigenetics "the soft" part. The epigenetic factors are a form of cellular memory obtained as a response to environmental variations and may influence the way and the duration of gene expression through an apparently simple mechanism of the "turn on" or "turn off" type. Three main answers can be given to the question in the title:

1. Because it calls attention to the importance of fetal development and childhood in the source of

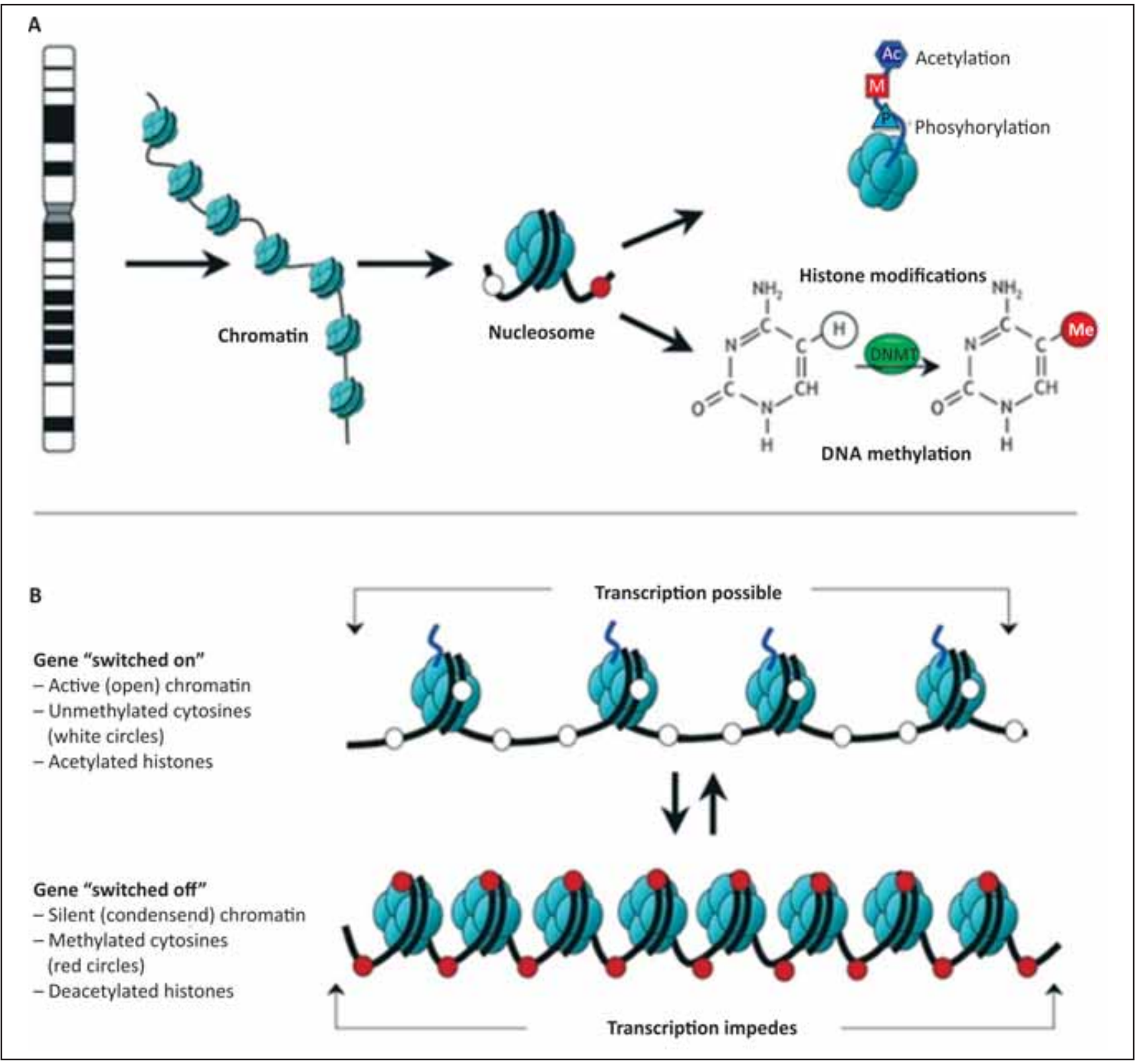

FIGURE 1. Epigenetic modifications of Modified DNA after Rodenhiser and Mann 2006 (2) 
some conditions which manifest during adulthood and old-age;

2. Because it argues that some of the parents' features are transmited to descendants through the memory of gametes, without the alteration of DNA sequence;

3. Because it emphasizes the permanent reorganization of cellular functioning and the way in which the environmental influences (nutritional, toxic, lifestyle) make a mark on pathology.

In recent years, genetic research (see GWASGenome-Wide Association Studies) has identified genes or genetic variations which are the basis of some diseases with complex transmission. The evolution of GWAS studies regarding the role of genetic factors in the development of some conditions was based on the association between genome variations and their phenotypic counterpart in populations. The "common disease", "common version" hypothesis states that adult common illnesses may be attributed to some allelic variants of common risk, present in at least $1-5 \%$ of the population. Nevertheless, the inborn character of complex adult disease was proven only in a relatively small amount $(25 \%)$. The decisive role of some genetic variants in common obesity, arteriosclerosis, type 2 diabetes is not convincingly backed up by statistical data, that is why the paradigm shift regarding the pathogenesis of these diseases has imposed a new component, the epigenetic one. Can epigentics, this new branch of genetics, offer explanations for some diseases that start in the intrauterine life and manifest themselves in adulthood? Can paediatrics be a key field in finding solutions to the epidemic development of some modern age diseases? Epigenetics explains the way in which the zygote that develops in over 220 cell types with the same DNA differentiates in cells and organs with different structure and functions. For most of the authors, the term of epigenetics refers to the epigenetic inheritance, i.e. to the transmission from one generation to the next of changes in gene expression, triggered by different stimuli. The epigenetic variations manifest in all life stages, being permanent modulators in response to toxicants, infections, changes in nutritional intake, carcinogenic noxae, socioemotional interactions (3). We are going to present some areas of pathology in which the epigenetic changes are at the forefront. Acute and chronic infections, allergic ailments, malignant diseases and adult metabolic conditions show a significant epigenetic dimension. The above-mentioned diseases in which epigenetics contributes to understanding of pathogenetic mechanisms may benefit from new therapies, some of which are already being implemented.

\section{Epigenetic changes produced by bacterial and viral infections}

Pathogenic germs adapt to their hosts and have the ability to interfere with the cellular and tissular processes, in a cell-to-cell war after surpassing the immune defence systems. The microbial manipulation of epigenetic mechanisms caused by intracellular parasites allowed these to develop numerous ways of taking substrates so as to complete their life cycle and to avoid the host's immune responses (4-6). The Helicobacter pylori (Hp) infection triggers an inflammatory process that may lead to chronic gastritis, gastric or duodenal ulcer. Chronic inflammation is followed by changes in the mucosa and sometimes malignization. Hp virulence factors are VacA and CagA cytotoxin. CagA oncoprotein acting in the gastric epithelial cells induces uncontrollable cell proliferation and chromosomal instability. Epigenetic changes related to carcinogenesis include both global genomic hypomethylation and hypermethylated regions which comprise tumor suppressor genes (4). Mycobacterium tuberculosis and other intracellular pathogens have developed numerous epigenetic mechanisms which inhibit IFN- $\gamma$ secretion and immunity-related gene transcription. Some bacterial species, among which we mention Shigella flexneri, Escherichia coli, Chlamydia pneumonia, are capable of producing epigenetic changes in human cells. Through their effector molecules, hepatitis viruses unbalance the progression of the cell multiplication cycle, and through their oncoproteins, produce global DNA hypomethylation, a phenomenon widely spread in neoplastic cells. Hepatocellular carcinomas of different viral etiology distinguish through a unique epigenetic "signature", specific viral (4).

\section{Epigenetic changes in allergic diseases}

The alarming rise in the incidence of some allergic conditions such as asthma, allergic rhinitis and eczema, was linked to environmental and lifestyle changes, suggesting that the functioning of the immune system is significantly influenced by them. Although the genetic component of asthma seems obvious in epidemiological studies, so far there has not been any focus on specific genes or the suggested genes have shown a variation in population. Recently, GWAS (Genome-Wide Association Studies) made possible the identification of some genes involved in inborn and adaptive im- 
mune response (7). The input of epigenetic mechanisms in allergic diseases was proven in clinical and experimental studies. They regard the fetal stage, significant in immune development, the differences in the expression of some genes in allergic diseases, the epigenetic regulation of immune development and the factors that modulate the allergic risk via an epigenetic way. Studies on asthmatic patients and monozygotic twins discordant for asthma shed light on aberrant DNA methylation, histone modifications with the programming of $T$ cell responses and other immune parameters as early as the intrauterine life stage (8-10). These changes will determine the risk for asthma and the severity of this disease in the later life stages. Although there is a strong connection between the genetic background and asthma, a study on monozygotic twins discordant for asthma has shown significant differences concerning the epigenetic changes (DNA methylation) of $\mathrm{T}$ cells for those affected by disease compared to non-asthmatic twins (11). (Fig. 2)

\section{Metabolic programming in children}

The first indications that the intrauterine environment in which the foetus develops has an important influence on health and survival date from the 90s. Correlations have been established between the low birth weight and later evolution towards obesity, HT, type 2 diabetes, cardiovascular diseases (12). Numerous studies involving scores of thousand of subjects have reached the conclusion that nutritional deficiency during pregnancy or noxae acting on the foetus during critical developmental periods result in the formation of an economic phenotype (i.e. programmed to consume less). Premature babies, small for gestational age or only affected by maternal nutritional restrictions during pregnancy, will later develop (sometimes from the adolescence) metabolic diseases that define adult or old age pathology. Under the name of fetal origins theory (13), the predictive adaptive re-

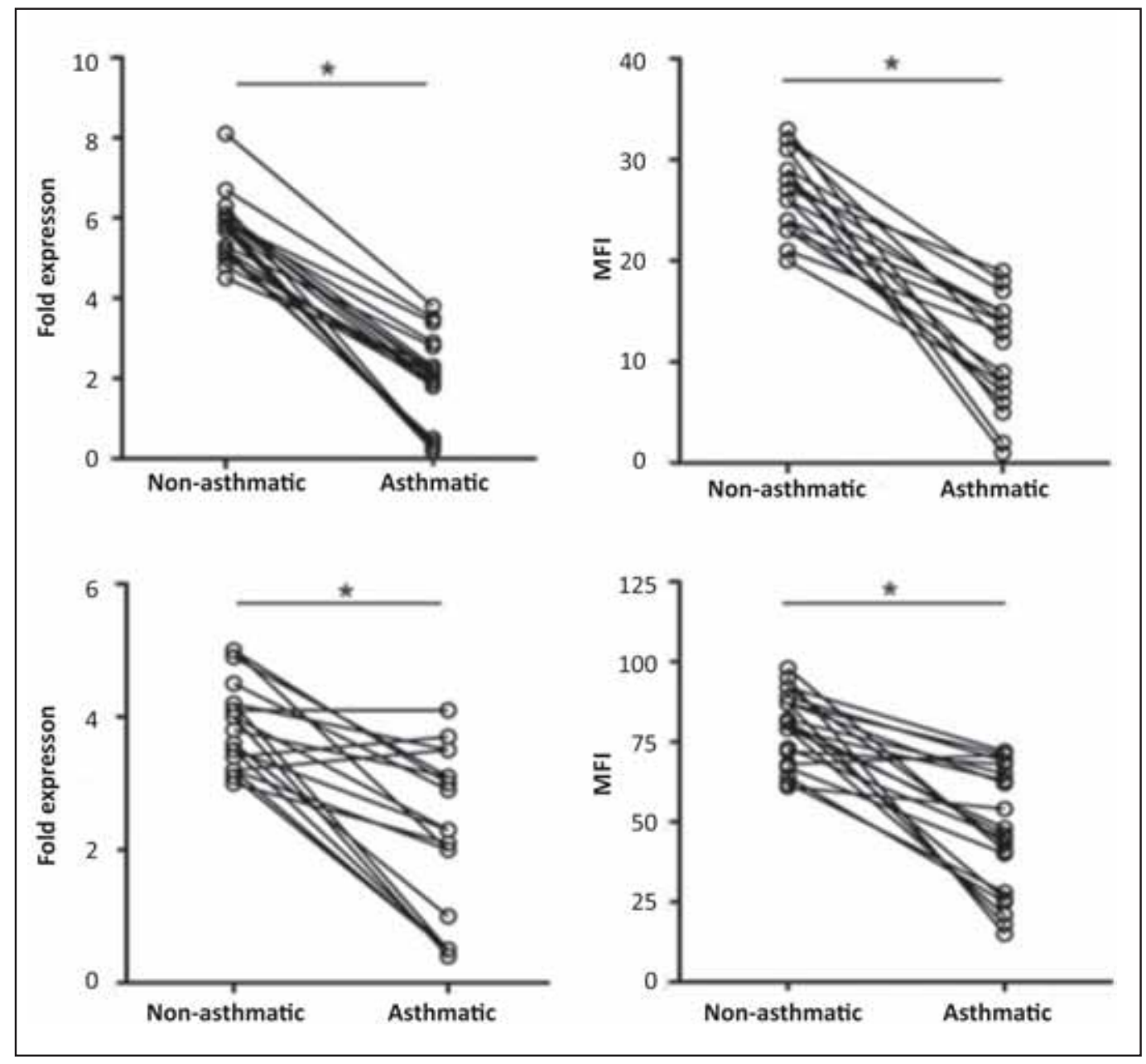

FIGURE 2. The decrease of proteins involved in effector T cells (Teff) and regulatory T cells (Treg) in monozytotic twins discordant for asthma.

A) FOXP3 expression (the protein involved in immune response regulates the development/ function of Treg cells); B) The level of FOXP3 protein extracted from Treg cells;

C) The level of IFN-y protein extracted from Teff cells after Runyon et al, 2012 (9) 
sponse $(14,15)$ or the developmental origins of health and disease $(16,17)$, are gathered clinical and epidemiological experimental data, which follow the installation stages of adult metabolic pathology and the molecular substrate of these modifications. A good example of the tardy effects of pregnancy malnutrition was represented by the famine age in Netherlands during World War II, when the effects of the fetal nutrition deficiencies were obvious in the adult pathology 50 years later (18). (Fig. 3)

The implications of intrauterine malnutrition have a major significance on the pahtology of wide geographical areas. Yajnick et al (19), taking into account that Indian babies are on average smaller than the European ones, has shown that their adiposity and hyperinsulinemia are present at birth. Comparatively, a group of 157 Indian babies weighing on average $2,800 \mathrm{~g}$, compared to $3,475 \mathrm{~g}$ in England, had higher insulin concentrations in the umbilical cord than the control group $(34.7 \mathrm{pmol} / 1$ VS $20.8 \mathrm{pmol} / \mathrm{l})$. Wang et al (20) have studied a cohort of children; some of them were full term babies and others with different degrees of prematurity. Plasma insulin levels were 2 times higher in babies with advanced prematurity compared to the full term babies and they maintained at a high level afterwards. Paradoxically, macrosomic babies or those with diabetic mothers have a pathologic trajectory convergent with those of premature babies or "small for the date" through the rise of plasma insulin, obesity and metabolic disease in adolescence and adulthood. Marceau et al (21) have compared the weight of children born before the maternal bariatric surgery in 783 obese women with other children delivered by women who went through the same type of surgery. From the 166 children born after surgery only $7.7 \%$ had fetal macrosomia compared to $34.8 \%$ in the maternal group which did not undergo this surgical procedure. These data have shown the relevance of maternal metabolic offer for fetal metabolism and its effects on the next generation, without the interference of some genetic factors. Changes in maternal and fetal metabolism contribute to programming of intrauterine development; this program structures the functioning of the organism in later life. Taking into account the growth rhythm of obesity in children in our country, we can expect negative effects on future generations.

\section{Epigenetic modifications in behavioral and psychiatric disorders}

The intervention of epigenetic processes in psychic processes is conditioned by family, social and environmental factors. Experimental research has shown that newborn animals carefully nursed by their mothers during the first week of life display modifications of gene expression at the cortical level and low responses to stress. These durable responses are different from those of the neglected babies that will disproportionately react to the aggressive environmental factors. Similar changes were distinguished among abused and neglected children, due to epigenetic modifications of the glucocorticoids in the hippocampus (22). Signals from the social environment contribute to the continuous remodeling of neuronal functions through the epigenetic apparatus, the processes of DNA methylation and chromatin configuration being active and dynamic during life (23). Unlike genetic mutations, cellular memory induced through epigenetic way is reversible, as shown by the evolution of modifications of obsessive/compulsive type,
First trimester

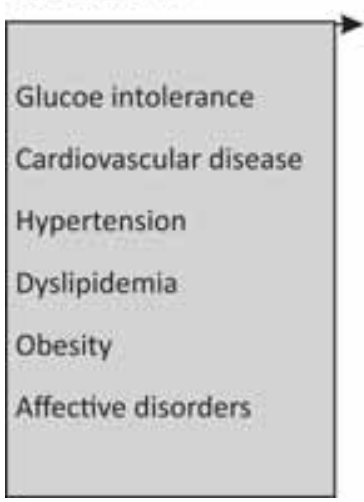

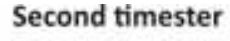

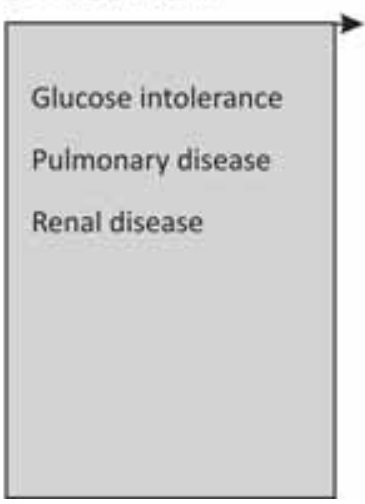

Third trimester

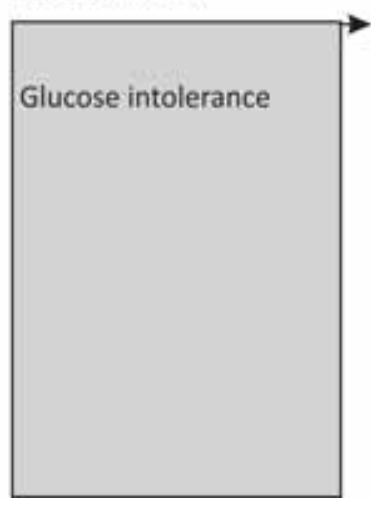

FIGURE 3. The structure of adult pathology of over 50 according to intrauterine malnutrition period during pregnancy, Netherlands 1944-1945 after Boekelheide et al 2012 (18) 
of depression or panic attacks (24). For the latter, modification in the emotional context, cognitive behavioral therapy and physical exercise contribute to the change of the metylation stage of some genes (e.g. monoamine oxidase [MAO]) and to the different expression of these (25).

\section{The epigenetic transgenerational transmission}

The installation of elements of the metabolic syndrome such as obesity and type 2 diabetes may constitute additive effects during many generations (26). Studies for more than 100 years on some Swedish families have shown that men who had wide access to food products during prepubertal age had grandchildren with high death risk of type 2 diabetes. Other studies have shown that mortality caused by diabetes and cardiovascular disease was influenced by the nutritional state of parents and grandparents. These studies support the idea that there is a life environment memory transmitted through gametes and reflected in the metabolic disorders of the descendants $(27,28)$. It was long thought that epigenetic modifications during lifetime are erased during gametogenesis, i.e. the spermatozoid and ovule will not bear the epigenetic experience acquired by the genitor. However, current data show that epigenetic markers are not fully disposed of at the passage between generations, and, as elements from the predecessors' experience, may pass to children, grandchildren and their descendants. Transgenerational epigenetic inheritance results in the formation of phenotypes in successive generations, as a result of epigenetic modifications transmitted through gametes (28). The preservation of the data offered by environment may be a functional adaptation mechanism but also a source of metabolic disorders. Some of the numerous examples of epigenetic transmission come from the experimental research in which nutritional imbalances from the first generation are sources of obesity, insulin resistance, type 2 diabetes and arteriosclerosis for the next generations (29-31). Transgenerational epigenetic inheritance erases the demarcation between the transmission based on DNA sequences and epigenetic transmission and, although it is another controversial notion, it changes the belief that complex chronic diseases are fully of genetic origin (31-33). The final destination of these studies consists in public health policies which have to cope with a wave of rapid changes in the nutrition and pathology state in different parts of the world (34).

\section{Epigenetic information sources for the medical practitioner}

In the absence of some widely available investigations of epigenetic markers, knowing some parental factors involved in the epigenetic pathology is useful. The main maternal factors linked to epigenetic pathology are: weight (BMI), excessive weight gain during pregnancy, age, smoking, nutritional restriction/excess, diabetes, HT, preeclampsia, asthma, allergic diseases, psycho-social stress, depression, traumas, professional/environmental exposure to chemicals (35). A series of factors with effects on adult pathology can be easily identified from neonatal period. Among these we mention as alarming factors: weight for gestational age (small for gestational age), prematurity, macrosomia, toxicants in neonatal period (35). Prophylactic approach, the cheapest therapy, is widely accessible. Is it possible that diet changing and elimination of weight excess of future mothers could have a long term influence on phenotype and the baby's health? Clinical trials have shown that after drastic decrease of obesity due to bariatric surgery maternal metabolic reprogramming and the decrease of child obesity have occurred. Obesity control on population level has proven a failure. In order to acquire a nutritional balance, a series of objectives have been set, among which we mention:

1. Nutritional approach of women in the periconceptional period through sanitary education for supporting fetal health;

2. Stimulation of natural nutrition;

3.Introduction of some elementary notions about adequate nutrition and its importance in the school curriculum;

4. Widespread insertion of sport and physical exercise into the education system;

5. Development of some governmental policies for consumers' protection regarding the trade of some high-fat and high carbohydrate content foods and high in salt foods through warning, differential labeling and permanent information. It is important that epigenetic flexibility allows the body to adapt rapidly to environmental fluctuations and this dynamic process can be influenced.

\section{Epigenetic therapy}

Some nutrients or food supplements have a biologic impact due to their intervention in the recovery of the epigenetic balance. Suggestion has been made that there are effects of polyphenols contained by green tea and red wine on diet regarding their antitumoral activity. (Table 1) 
TABLE 1. Nutritional nutrients/supplements with epigenetic effects partially after Szyc et al, 2015 (36)

\begin{tabular}{|l|l|}
\hline Nutritional frame & Effectors \\
\hline Methyl donors & $\begin{array}{l}\text { Folate, choline, vitamin B12, methionine, } \\
\text { betaine, serine, glycine }\end{array}$ \\
\hline Fatty acids & DHA, EPA, ARA, butyrate \\
\hline Vitamine & Vitamin D, vitamin C, retinol, tocopherols \\
\hline Phyto-derivatives & $\begin{array}{l}\text { Resveratrol, curcumin, polyphenols, } \\
\text { genistein, soy isoflavones }\end{array}$ \\
\hline
\end{tabular}

The most significant promise of epigenetic therapy is connected to cancer. It was believed that cancer inception is caused by the accumulation of genetic abnormalities due to mutations, deletion, translocation processes, which allow the development and proliferation of malignant cells. Moreover, there have been noticed epigenetic disorders through DNA methylation, histone modifications

\section{REFERENCES}

1. Smith R., Mill J. Epigenetics and Chronic Diseases: An Overview, in Roach $\mathrm{H}$ et al (Eds): Epigenetic Aspects of Chronic Diseases Springer_Verlag, 2011

2. Rodenhiser D., Mann M. Epigenetics and human disease: translating basic biology into clinical applications. CMAJ, 2006,174, 3, 341-348

3. Stein R., Davis D. Epigenetics: A Fascinating Field with Profound Research, Clinical, \& Public Health Implications. Amer Biol Teacher, 2012, 74, 4, 213-223

4. Niller H., Minarovitz J. Epigenetics and Human Infectious Diseases in Tollefsbol (Ed): Epigenetics in Human Disease. Elsevier Inc, 2012

5. Akeel R. Role of epigenetic reprogramming of host genes in bacterial pathogenesis. Saudi J Biol Sci, 2013, 20, 305-309

6. Gomez-Diaz E., Jorda M., Peinaldo M. et al. Epigenetics of Host-Pathogen Interactions: The Road Ahead and the Road Behind. PLOS Pathogens, 2012, DOI:10.1371/journal.ppat.1003007

7. Portelli M., Hodge E., Sayers I. Genetic risk factors for the development of allergic disease identified by genome-wide association. Clin Exp Allergy, 2015, 45, 1, 21-31

8. Shuk-Mei H. Environmental Epigenetic of Asthma-An update. J Alllergy Clin Immunol, 2010,126, 3, 453-465

9. Lovinski-Desir S., Miller R. Epigenetics, Asthma, and Allergic Diseases: A Review of the Latest Advancements. Curr Allergy Asthma Rep, 2012, 12, 3, 211-220

10. Amarasekera M., Martino D., Tulic M. et al. Epigenetic Aberrations in Human Allergic Diseases, In Tollefsbol (Ed): Epigenetics in Human Disease. Elsevier Inc, 2012

11. Runyon R., Cachola L., Rajeshuni N. et al. Asthma discordance in twins is linked to epigenetic modifications of T cells. PLOS One, 2012, 7, 11, e48796

12. Simmons R. Devlopmental Origins of Diabetes: The Role of Epigenetics. In J.R. Seckl and Y Christen (eds), Hormones, Intrauterine Health and Programming, Springer 2014

13. Barker D.J.P. Fetal origins of coronary heart disease. BMJ 1995, 311, 171-174

14. Gluckman P.D., Hanson M.A. Metabolic Disease: Evolutionary, Developmental and Transgenerational Influences in Hornestra G., Uauy R., Yanf X. (eds): The Impact of Maternal Nutrition on the Offspring. Nestle Nutrition Workshop Series Pediatric Program, 2005, 55, 17-27

15. Gluckman P., Low F., Buklijas T. et al. How evolutionary principles improve the understanding of human health and disease. Evolutionary Applications Blakwell Publishing Ltd 2011, 4, 249-263 and of microARN with the expression of the genes that transform normal cells in malignant cells (37). Unlike genetic mutations, epigenetic modifications are reversible and may reactivate the antitumoral cell systems or may inhibit oncogenic transcription. Based on this concept, new therapy strategies are being developed and some are made clear. $\mathrm{Nu}-$ trition in all life stages, stress, physical activity, smoking, alcohol, infections can destabilize epigenetic markers encouraging metabolic disease and cancer. Epigenetic principles of sanogenesis must be put into practice from pregnancy and must be monitored throughout childhood as a premise for a future good health. Far from being a discovery in the search of some applications, epigenetics represents a great chance to improve medical paradigms.

16. Langley-Evans S., McMullen S. Developmental origins of adult disease. Med Princ Pract, 2010, 19, 87-98

17. Langley-Evans S. Nutrition a lifespan approach. Wiley-Blackwell, 2009

18. Boekelheide K., Blumberg B., Chapin R., Cote I. Predicting Later-Life Outcomes of Early-Life Exposures. Environ Health Persp, 2012, 120, 10, 1353-1361, 12, 60(suppl 3), 38-43

19. Yajnick C., Lubree H., Rege A. et al. Adiposity and Hyperinsulinemia in Indians Are Present at Birth. J Clin Endocrinol Metab, 2002, 87, 12, 5575-5580

20. Wang G., Divall S., Radovick S. et al. Preterm birth and random plasma insulin levels at birth and in early childhood. JAMA, 2014, 311, 6, 587-596

21. Marceau P., Kaufman D., Biron S. et al. Outcome of pregnancies after biliopancreatic diversion. Obes Surg, 2004, 14, 3, 318-324

22. McGowan P., Saasaki A., D'Alessio A. et al. Epigenetic regulation of the glucocorticoid receptor in human brain associates with childhood abuse. Nat Neurosci 2009, 12, 3, 342-348

23. Szyf M., McGowan P., Meaney M. The Social Environment and the Epigenome, Environ Molec Mutagen, 2008, 49, 46-60

24. Mostafavi-Abdolmaleki H., Glatt S., Tsuang M. Epigenetics in Psychiatry in Roach $\mathrm{H}$ et al (Eds): Epigenetic Aspects of Chronic Diseases Springer-Verlag London 2011

25. Ziegler C., Richter J., Mahr M. et al. MAOA gene hypomethylation in panic disorder-reversibility of an epigenetic risk pattern by psychotherapy. Trans/ Psychyatry, 2016, Apr 5, 6, e773

26. Rockholm B., Baker J., Sorensen T. The levelling off the obesity epidemic since the year 1999 - a review of evidence and perspectives. Obes Rev, 2010, 11, 12, 835-846

27. Manor O., Koupil I. Birth weight of infants and mortality in their parents and grand parents: the Uppsala Birth Cohort Study. Intern J Epidemiol, 2010, 39, 1264-1276

28. Kaati G., Bygren L., Edvinsson S. Cardiovascular and diabetes mortality determined by nutrition during parent's and grandparent's slow growth period. Eur J Hum Genet, 2002, 10, 11, 682-688

29. Barres R., Zierah J. DNA methylation in metabolic disorders. Am J Clin Nutr, 2011, doi: 10.3945/ajcn.110001933

30. Lillycrop K., Phillips E., Torrens C. et al. Feeding pregnant rats a protein-restricted diet persistently alters the methylation of specific cytosines in the hepatic PPARa promoter of the offspring. Br J Nutr, 2008, 100, 2, 278-282

31. Lillycrop K., Burdge G. Epigenetic changes in early life and future risk of obesity. Intern J Obesity, 2011, 35, 83 
32. Waterland R., Jirtle R. Early Nutrition, Epigenetic Changes at Transposons and Imprinted Genes, and Enhanced Susceptibility to Adult Chronic Diseases. Nutrition 2004, 20, 63-68

33. Smith R., Mill J. Epigenetics and Chronic Diseases: An Overview, in Roach $\mathrm{H}$ et al (Eds): Epigenetic Aspects of Chronic Diseases Springer_Verlag, 2011

34. Niculescu M. Epigenetic transgenerational inheritance: Should obesity-prevention policies be reconsidered ? Synesis: J Sci Tehnol Ethics Policy, 2011
35. Wright R., Saul R. Epigenetics and Primary Care, Pediatrics, 2013, 132, S216-S223

36. Szic K., Declerck K., Vidakovic M. et al. From inflammaging to healthy aging by dietary lifestyle choices: is epigenetics the key to personalized nutrition? Clin Epigen, 2015, 7, 33 DOI: 10.1186/ s13148-015-0068-2

37. Hatzimichael E., Crook T. Cancer Epigenetics: New Therapies and New Challenges. J Drug Delivery, 2013, ID 529312 